\section{What is lost in translation?}

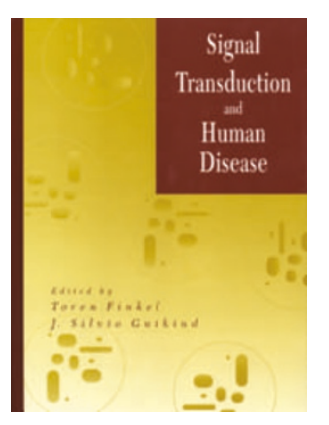

\section{Signal Transduction and Human Disease}

\author{
edited by Toren Finkel and \\ J. Silvio Gutkind
}

John Wiley \& Sons • 2003

Hardback, £64.50/\$99.95

Henry R. Bourne

How well are biomedical scientists translating advances of molecular and cell biology into practical treatments for common diseases? The editors of Signal Transduction and Human Disease deliver a cautious verdict: "Although it is too early to know for sure, certain promising signs are emerging." Their book shows that the promise of 'translational' research is real and the caution warranted.

The editors, Toren Finkel and Silvio Gutkind, have assembled 14 chapters, each focused on molecular approaches to devising therapy for a common disease. Topics include coronary artery disease, cancer, adult-onset diabetes, neurodegenerative disorders and rheumatoid arthritis, with a final chapter on drug development and high throughput screening. Unable to find any "single text that straddle(s) the... interface between biology and modern medicine," the editors asked authors to provide clinical descriptions of the disease (for scientists) and accounts of the relevant biology and strategies for tackling potential therapeutic targets (for clinicians).

Unfortunately, many chapters fail at one or both tasks. Many clinical descriptions are not very informative. Can one begin to think clearly about adult-onset diabetes without first recognizing that reducing excess intake of carbohydrate and calories ameliorates the symptoms of many patients, or about cancer with barely a nod towards the genomic instability that allows cancers to select ever-more malignant phenotypes from a cornucopia of gene mutations, deletions and duplications? Conversely, several detailed accounts of biological regulation will induce terminal boredom in a bright, well-informed clinician (prediction) or acute intellectual indigestion in an investigator of signal transduction (observation). Because each chapter stands independent of all the others, as in a symposium volume, the reader is treated to multiple accounts of JAK/STAT signalling, small GTPases, MAPK cascades, NFK-B and so on.

The real culprits are not the editors, nor the authors, however. Instead, this book accurately mirrors the inadequacy of our current

Henry R. Bourne is in the Departments of Cellular and Molecular Pharmacology and Medicine, University of California School of Medicine, San Francisco, CA 94143, USA.

e-mail: bourne@cmp.ucsf.edu knowledge in the face of daunting complexity. Faced with questions to which they don't know the answers, most contributors - like most of us, most of the time - choose to cover a manageable sub-topic rather than the disease itself: oxidative stress at vascular endothelia rather than coronary artery disease, NFK-B signalling rather than asthma, mutations of a cytokine receptor subunit rather than severe combined immunodeficiency, and tumor necrosis factor $\alpha$ signalling rather than rheumatic diseases.

Most chapters give short shrift to the issue of complexity itself, including genetic and other causes of disease, and concentrate instead on downstream pathogenic mechanisms. The two cancer chapters cite blizzards of mutations that enhance proliferation or inhibit apoptosis, each considered as if it functioned alone to cause cancer. Instead, we know that most cancers result from different combinations of two, three, or many mutations. If so, what determines which mutant gene or aberrant regulatory pathway is a potentially attractive target for therapy of an individual cancer? What makes chronic myelogenous leukemia almost uniquely susceptible to treatment with Gleevec, a selective tyrosine kinase inhibitor?

Similarly, discussions of adult-onset diabetes, arthritis, coronary artery disease, Alzheimer's disease and other conditions cite examples of inherited mutations that increase susceptibility to disease, but ignore or barely touch on multi-gene pathogenesis and environmental factors (for example, diet, oxidative stresses and exposure to antigens) that combine with mutations to modify the clinical severity and outcome of diseases in individual patients. Nonetheless, these variables certainly affect the likelihood of developing an effective drug aimed at a single protein target.

One valid response to such criticisms is that it is often more useful to focus on pathogenic mechanisms, which we may understand and can do something about, than on the (usually) unknown causative mutations in an individual patient. Nonetheless, such answers may sometimes lead us astray. An example: although resistance to the glucose-lowering effects of insulin is important for the pathogenesis of adult-onset diabetes, the mix of causative mutations in some patients probably reflect beneficial phenotypes selected in evolution when the human diet was less rich than it is now in developed countries. Consequently, such patients may benefit much more from dietary restriction and consequent weight loss than from administration of insulin or drugs that increase insulin secretion.

Although clear successes are few, this book does recount in detail several well-studied examples of approaches that promise to convert molecular knowledge into clinical usefulness. Scientists already engaged in this effort will already know many of the stories. Clinicians may find that the book tells them more than they want to know. Nonethless, students may find chapters useful as introductions to particular diseases. Signal Transduction and Human Disease presents accurate snapshots of our knowledge and ignorance at the beginning of the twenty-first century. Although such a photo album can be valuable, I suspect that a more analytical approach, focusing on principles rather than examples, could have produced a more useful book. 